\title{
QoS-Aware Energy Management in Body Sensor Nodes Powered by Human Energy Harvesting
}

\author{
Ernesto Ibarra, Member, IEEE, Angelos Antonopoulos, Senior Member, IEEE, \\ Elli Kartsakli, Senior Member, IEEE, Joel J. P. C. Rodrigues, Senior Member, IEEE, \\ and Christos Verikoukis, Senior Member, IEEE
}

\begin{abstract}
Harvesting energy in the human environment has been identified as an effective way to charge the body sensor nodes in wireless body area networks (WBANs). In such networks, the capability of the nodes to detect events is of vital importance and complements the stringent quality of service (QoS) demands in terms of delay, throughput, and packet loss. However, the scarce energy collected by human motions, along with the strict requirements of vital health signals in terms of QoS, raises important challenges for WBANs and stresses the need for new integrated QoS-aware energy management schemes. In this paper, we propose a joint powerQoS (PEH-QoS) control scheme, composed of three modules that interact in order to make optimal use of energy and achieve the best possible QoS. The proposed scheme ensures that a sensor node is able to detect the medical events and transmit the respective data packets efficiently. Extensive simulations, conducted for different human activities (i.e., relaxing, walking, running, and cycling), have shown that the application of PEH-QoS in a medical node increases the detection efficiency, the throughput, and the energy efficiency of the system.
\end{abstract}

Index Terms-WBAN, quality of service, energy harvesting, wireless sensor networks, e-health.

\section{INTRODUCTION}

\section{A. Motivation}

W IRELESS Body Area Networks (WBANs) face several challenges that should be overcome before their f nal implementation in telemedicine systems. The medical devices that form the network, called Body Nodes (BNs), are generally heterogeneous, performing distinct tasks and having different power supply and Quality of Service (QoS) requirements.

Manuscript received August 28, 2015; accepted September 18, 2015. Date of publication September 28, 2015; date of current version December 22, 2015. This work was supported in part by the Agència de Gestió d'Ajuts Universitaris i de Recerca Project under Grant 2014-SGR-1551, in part by the Wireless Sensor Networks for Quality of Life Project under Grant 286047, in part by the Instituto de Telecomunicações, NetGNA Group, Covilhã Delegation, and in part by the National Funding through the Fundação para a Ciência e a Tecnologia under Project UID/EEA/500008/2013. The associate editor coordinating the review of this paper and approving it for publication was Dr. Roozbeh Jafari.

E. Ibarra is with the University of Barcelona, Barcelona 08007, Spain (e-mail: e.ibarra@ieee.org).

A. Antonopoulos and E. Kartsakli are with the Department of Signal Theory and Communications, Technical University of Catalonia, Barcelona 08034, Spain (e-mail: angelos.antonopoulos@tsc.upc.edu; ellik@tsc.upc.edu).

J. J. P. C. Rodrigues is with the Instituto de Telecomunicações, University of Beira Interior, Covilhã 6201-001, Portugal, and also with the University of Fortaleza, Fortaleza 60811-905, Brazil (e-mail: joeljr@ieee.org).

C. Verikoukis is with the Telecommunications Technological Center of Catalonia, Barcelona 08860, Spain (e-mail: cveri@cttc.es).

Color versions of one or more of the fgures in this paper are available online at http://ieeexplore.ieee.org.

Digital Object Identifie 10.1109/JSEN.2015.2483064
More specificall, the QoS can be define in terms of delay, throughput and packet loss, while the vital functions performed by the BNs make the detection of medical events extremely crucial for the human/patient monitoring.

In addition, these networks usually face space constraints in order to adapt seamlessly to the human body, limiting the number and the size of the nodes in the network. The $\mathrm{BN}$ dimensions are strongly related to the battery's weight and size, which is proportional to the battery capacity. Since the battery is a f nite source of energy, as the battery level drops, the BN operation becomes compromised and eventually stops. To resume operation, it is necessary to replace or recharge the battery as soon as possible. However, battery replacement is not always feasible, since it might damage the $\mathrm{BN}$ and even jeopardize the patient's health. This problem is exacerbated in the case of implantable nodes, where $\mathrm{BN}$ replacement would require surgical procedures [1]. Increasing the battery capacity could prolong the $\mathrm{BN}$ lifetime, however, this solution is not viable in WBANs [2], since it would lead to an increase in the $\mathrm{BN}$ dimensions and weight.

Harvesting energy from other available sources [3] could permanently supply the node with the required power, providing the most promising solution to the power supply problem. Using special hardware devices, known as energy harvesters, BNs can convert various types of energy (e.g., heat, motion, etc.) [4] into electrical energy. The use of Energy Harvesting $(\mathrm{EH})$ combined with a rechargeable storage device (e.g., a battery or a supercapacitor) can provide autonomous $\mathrm{BN}$ operation. However, $\mathrm{EH}$ introduces an additional variable to the network, which is related to the availability and quantity of the collected energy. Energy harvesters in the human environment provide a smaller and time-dependent amount of energy with respect to the batteries. Hence, the successful exploitation of the collected energy is a key factor for the smooth BN operation. In this context, Kansal et al. [5] introduced the concept of Energy Neutral Operation (ENO) to achieve infi ite lifetime and perpetual operation, as long as there are no hardware failures. More specif cally, a node powered by energy harvesting is declared to be in ENO state if it consumes less or equal amount of energy compared to the energy harvested from the environment [5].

In the context of WBANs, it is also possible to harvest energy from sources related to the human body, including mechanic, kinetic, thermal and biochemical sources [6], [7], a concept referred to as Human Energy Harvesting (HEH). However, in such scenarios, the problem of energy shortage 
is further exacerbated due to the low energy collection by the human motion. A promising solution is based on the piezoelectric harvester that converts the vibrations caused by the body movements into electrical energy [6]. Through appropriate power management circuits, such harvesters can continuously accumulate small amounts of energy for a long period and provide higher output power in a very short time [8], [9], taking full advantage of the human movements. Piezoelectric harvesters can also be combined with other types of EH sources (electromagnetic, solar, etc.), in order to increase the collected amount of energy [10].

Apparently, the scarce energy collected by human motions, along with the strict requirements of vital health signals in terms of QoS, raise important challenges for WBANs. Although many works on sensor networks study the QoS aspects of the communication [11], [12], significan research effort ([13]-[16]) has been recently devoted to the design of schemes that focus on the communication, taking also into account the energy harvesting conditions in the network. However, despite their novelty, the aforementioned works focus on specifi parameters and metrics (e.g., packet loss, event detection, etc.), neglecting the need for holistic approaches that respect all the applications requirements in WBANs.

\section{B. Contribution}

In this paper, we introduce a Power-QoS control scheme (PEH-QoS), designed for BNs operated by HEH. $\mathrm{PEH}-\mathrm{Q}$ S is an ENO inspired algorithm, developed for providing the best possible QoS under energy harvesting conditions. More specif cally, the proposed scheme:

- Promotes the detection ability of medical events (either normal or critical) through an ENO-based power management.

- Prevents the data queue saturation and maintains the clinical validity of the stored information.

- Improves the energy usage through a sophisticated module that enables the optimum packet aggregation in energy harvesting conditions.

To assess the performance of the proposed scheme in realistic HEH conditions, we have performed extensive simulations considering four different activities (i.e., relaxing, walking, running, cycling), corresponding to different rates of $\mathrm{EH}$.

The remainder of this paper is organized as follows. In Section II, we brief y review the background on the human energy harvesting and the related to our topic work. In Section III, we describe the BN architecture and the network topology and we provide details with regard to the EH model. Section IV introduces the PEH-QoS control scheme. In Section $\mathrm{V}$, we evaluate the performance of PEH-QoS by extensive simulations. Finally, Section VI concludes the paper.

\section{Related WORK}

The wide explosion of WBANs along with the proliferation of energy harvesting techniques have motivated several researchers to study the communication performance of WBANs in energy harvesting conditions. Seyedi and Sikdar [13] have conducted a modeling and

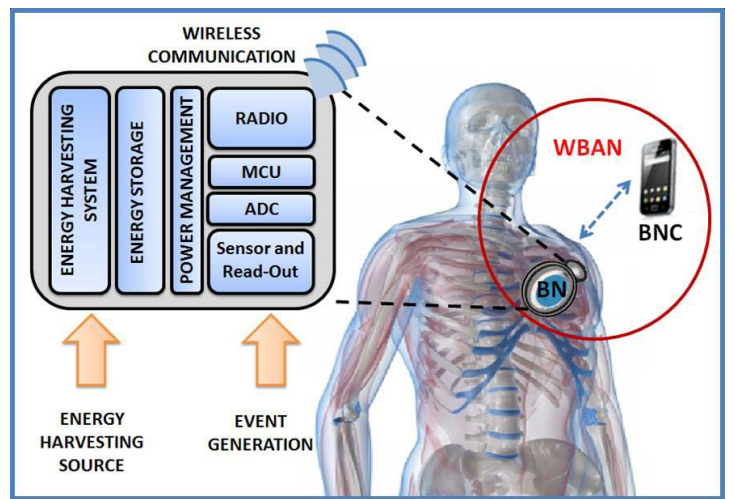

Fig. 1. System Model.

analysis of BN powered by energy harvesting in WBANs, by providing a discrete time model based on a two-state Markov chain, which integrates models for energy harvesting and $\operatorname{traff} \mathrm{c}$ generation. The aforementioned study is focused on fi ding the average probability that an event is not detected or transmitted (i.e., lost event), due to the lack of suff cient energy in the BN. In [14], the same authors have proposed a set of adaptive transmission policies, formulated as a Markov decision process, aiming to maximize the probability of event detection and transmission. The proposed schemes exploit information on the current BN energy level, the battery recharging state, as well as the event generation process.

Ventura and Chowdhury [15] have extended the research carried out in [13] and [14] by proposing a multiple board Markov model for energy harvesting sensors (MAKERS) that allows for the estimation of important performance metrics in HEH-WBANs. He et al. [16] have extended the above research by proposing analytical solutions for the optimal resource allocation to provide QoS guarantee to data delivery for the HEH-WBANs. The main goal of the proposed optimization schemes is to provide a sustainable QoS that guarantees low delay and low packet loss to subscribers.

All these works study particular aspects of the problem. Moreover, they show the operation degradation at the nodes due to EH, not only in packet transmission but also in the event detection. The time required to store the amount of energy needed to detect or report (transmit) an event depends on the amount of the collected energy in a given period of time. Based on the above, the main challenge in HEH-based WBANs is to ensure the correct event detection by the $\mathrm{BN}$ and guarantee the QoS in terms of delay, throughput and packet loss, which will be the key focus of our work.

\section{System ModeL}

\section{A. BN's Architecture and WBAN Topology}

We consider the WBAN topology depicted in Fig. 1. We adopt a Harvest-Store-Use architecture (i.e., the energy is stored in a supercapacitor before use) [17], and the WBAN is conf gured in a star topology with direct connection to the Body Node Coordinator (BNC). ${ }^{1}$ In addition, we

\footnotetext{
${ }^{1}$ Please note that, in our study, we assume a single BN in order to focus on the benef ts of the proposed scheme in ideal conditions (i.e., no contention in the channel access).
} 


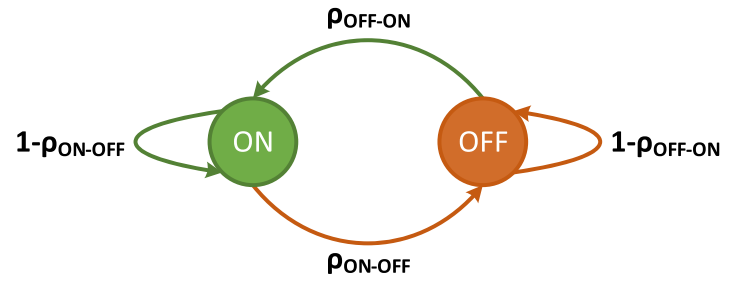

Fig. 2. Markov Chain: Availability of EH Source.

employ a $1.9 \mathrm{~nJ} / \mathrm{b}$ 2.4GHz Multi-standard (Bluetooth Low Energy/Zigbee/IEEE 802.15.6) transceiver for Personal/Body Area Networks [18], while the sensor and the reader are modeled based on a $30 \mu \mathrm{W}$ analog signal processor integrated circuit for biomedical signal monitoring [19].

In our model, the BNC is the network sink, responsible for setting up the WBAN and collecting all information transmitted by the BN. The data communication between the BNC and the $\mathrm{BN}$ takes place via a contention-free scheme, where the $\mathrm{BNC}$ polls the $\mathrm{BN}$ every predef ned time intervals according to the IEEE 802.15.6 polling access mode [20]. We assume that the $\mathrm{BNC}$ is a smartphone with high processing capabilities and an external power supply (i.e., unlimited power). On the other hand, the $\mathrm{BN}$ has an energy harvester able to collect the energy available in the human body. The BN's power consumption is divided into two main parts: $i$ the detection power consumption $\left(P_{d e t}\right)$, which includes the power consumption related to the correct BN's operation (i.e., microcontroller unit (MCU), analog-to-digital converter (ADC), sensor and read-out), and $i i$ the transmission power consumption $\left(P_{t x}\right)$, which includes the power consumption related to the duty cycle of the transceiver (i.e., data communication process).

\section{B. Energy Harvesting Model}

The availability of the harvesting source and the exploitation of the harvested energy constitute the two key factors that determine the ENO of a given system. In our case, we consider that the $\mathrm{BN}$ is equipped with a piezoelectric harvester, converting the vibrations caused by the body movements into electrical energy [6]. In our system model, time is divided into slots of duration $t_{\text {slot }}$. In each time slot, the harvester captures different amounts of power in the range of $P_{E \text { Hmin }}$ and $P_{E \text { Hmax }}$, denoting the minimum and the maximum amounts of power that can be harvested, respectively, according to the intensity of the movement. These power limits also vary for different activities (e.g., walking, relaxing, cycling, etc.). The frequency of movement disturbance is another important factor that should be taken into account. To model this physical phenomenon, we use a simple discretetime Markov chain with two states: the active state (ON) and the inactive state (OFF), as shown in Fig. 2.

In the ON state, human movements may occur, while in the OFF state, there is no significan movement that produces disturbances in the harvester (i.e., no energy is generated). Moreover, we defi e $\rho_{O F F-O N}$ as the transition probability from state OFF to state $\mathrm{ON}$, whereas $\rho_{O N-O F F}$ is define as the transition probability from state ON to state OFF. Therefore, the probabilities of remaining at the states $\mathrm{ON}$ and OFF are given by $1-\rho_{O N-O F F}$ and $1-\rho_{O F F-O N}$, respectively.

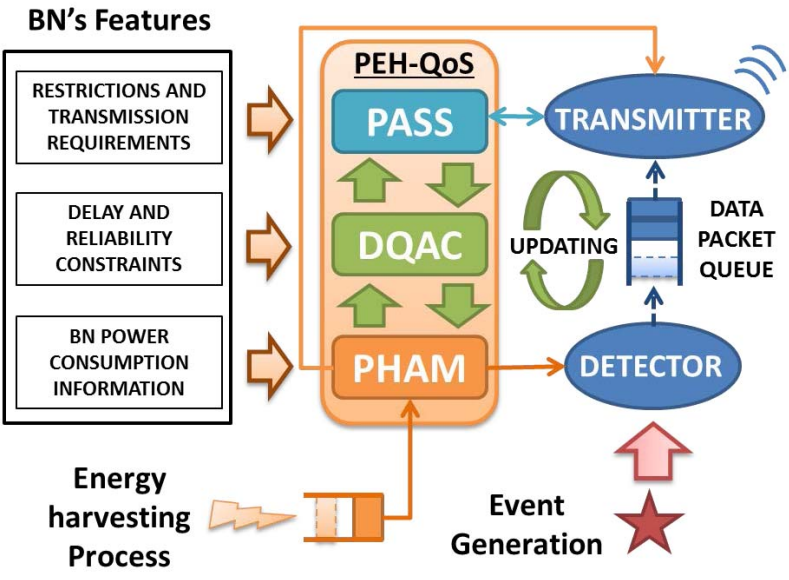

Fig. 3. PEH-QoS modules.

As a result, the steady state probabilities of the system are given $b^{2}$ :

$$
\begin{aligned}
\mu_{O N} & =\frac{\rho_{O F F-O N}}{\rho_{O N-O F F}+\rho_{O F F-O N}} \\
\mu_{O F F} & =\frac{\rho_{O N-O F F}}{\rho_{O N-O F F}+\rho_{O F F-O N}}
\end{aligned}
$$

Having formulated the body movement with the above Markov process, we can estimate the amount of harvested energy $E_{E H}(t)$ in one time slot as:

$$
E_{E H}(t)=P_{E H}(t) \cdot t_{\text {slot }},
$$

where $P_{E H}(t)$ is the power harvested in $t_{s l o t}$. More specifically, in active time slots (i.e., where motion is detected), $P_{E H}(t) \in\left[P_{E H \text { min }}, P_{E H \text { max }}\right]$, whereas in inactive slots, no energy is collected (i.e., $P_{E H}(t)=0$ ).

\section{Power-QoS Aware Management CONTROL SCHEME (PEH-QoS)}

The proposed scheme, PEH-QoS, is executed at each $\mathrm{BN}$, aiming to adapt the node's performance to its particular features, power supply and QoS requirements. To that end, PEH-QoS combines three interconnected modules, illustrated in Fig. 3: i) the Power-EH Aware Management (PHAM), which calculates and manages the harvested energy, aiming to control the overall energy consumption of the system and keep the node in ENO state, ii) the Data Queue Aware Control (DQAC), which manages the packet queue and ensures that only useful data are transmitted, by discarding any packets that have lost their clinical validity according to the delay and reliability requirements of the respective medical application, and iii) the Packet Aggregator/Scheduling System (PASS), which uses the amount of power available for transmission (information from the PHAM module) and the amount of data stored in the queue (information from the DQAC module) to determine the maximum number of packets that can be transmitted in each data communication process. In the following sections, we describe the operation of each module in detail.

\footnotetext{
${ }^{2}$ We are particularly interested in the probability $\mu_{O N}$, which represents the portion of time that energy is collected by the harvester.
} 


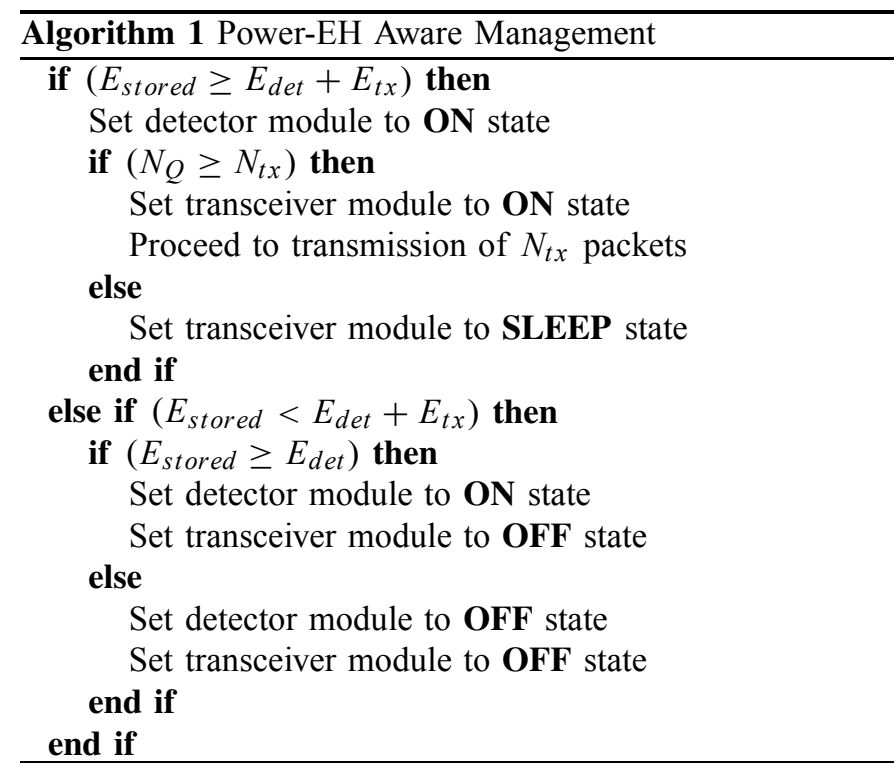

\section{A. PHAM: Power-EH Aware Management}

The PHAM module is responsible for the management of the harvested energy at the $\mathrm{BN}$, which plays a critical role to the system's performance. PHAM uses the BN's power consumption information to distribute the harvested energy among the two key tasks of the node, namely, event detection and packet transmission. Typically, the energy consumed by the radio module of the $\mathrm{BN}$ is much higher compared to the energy required by the detector, i.e., $E_{t x} \gg E_{d e t}$. Nevertheless, in WBANs, the detection capability of the BN can sometimes be more important than the transmission function, especially in the case of critical events.

Hence, the purpose of PHAM module is to eff ciently manage the available energy in order to maximize the number of detected events and maintain the node operation in ENO state. To guarantee a high rate of detected events, the PHAM module must ensure that the detector is provided with suff cient energy (i.e., at least $E_{d e t}$ ). On the other hand, in order to achieve the ENO, the consumed energy should not exceed the total available energy of the BN. The level of energy stored in the energy buffer ( $\left.E_{\text {stored }}\right)$ at a given time $t$ depends on the energy harvested from the human environment $\left(E_{E H}(t)\right)$, based on the following equation:

$$
E_{\text {stored }}(t)=E_{\text {stored }}(t-1)+E_{E H}(t) .
$$

The PHAM module is described in Algorithm 1. The algorithm is applied at the BN to control the power consumption based on the stored energy level. Its frst priority is to ensure the proper operation of the detector (i.e., $E_{\text {stored }} \geq E_{d e t}$ ), as the transmissions take place only when the energy level reaches $E_{\text {stored }} \geq E_{d e t}+E_{t x}$ (i.e., the available energy is suff cient for both transmission and detection). The amount of packets to be transmitted in each communication process, define as $N_{t x}$, and the energy required for their transmission $E_{t x}$ are calculated by the PASS module. Finally, it should be mentioned that if the number of packets stored in the data queue $\left(N_{Q}\right)$ is less that the optimum number of packets to be transmitted $\left(N_{t x}\right)$, the transceiver enters into

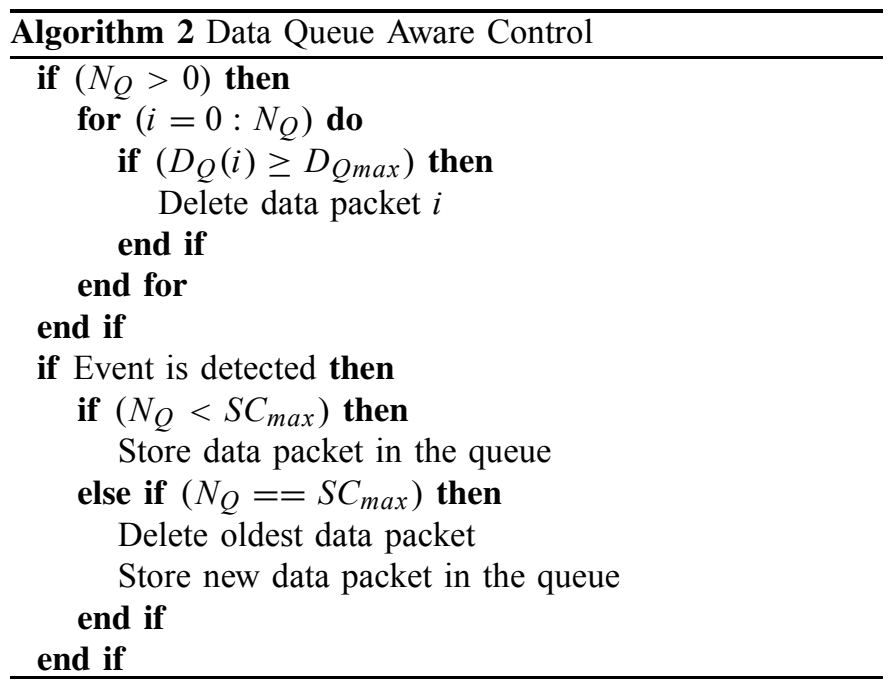

sleep mode, until suff cient packets are accumulated in the queue.

\section{B. DQAC: Data Queue Aware Control}

Since the amount of harvested energy depends on the availability of the EH source, data packets may remain stored for a long time before their transmission. This raises two major issues. The fi st is related to the saturation of the data queue, since the node has a f nite storage capacity. Once the buffer reaches its maximum capacity, it will not be able to store subsequent detected events and, consequently, these packets will be lost. The second problem is related to the loss of clinical validity of the stored data due to the queuing delay (e.g., in monitoring of vital signals, old data may lose their value in the presence of most recent events). DQAC is a module designed to control and manage the data queue by: i) preventing the saturation (overf ow) of the queue, by removing packets that lost their validity, and ii) allowing all detected events to be stored.

The DQAC module is described in Algorithm 2. DQAC proceeds to discard outdated packets and update the data queue using the information of the maximum allowed end-to-end delay $\left(D_{\max }\right)$, which is determined by the medical application requirements, and the maximum storage capacity $\left(S C_{\max }\right)$, which is a physical restriction of the BN's hardware. DQAC constantly monitors the waiting time of each data packet in the queue $\left(D_{Q}\right)$ and the number of stored packets $\left(N_{Q}\right)$. Given the time $T_{t x}$ that is required for the data communication process (calculated by the PASS module), the maximum waiting time of a packet in the queue $\left(D_{Q \max }\right)$ in order to satisfy the $D_{\max }$ QoS requirement is calculated as $D_{Q \max }=D_{\max }-T_{t x}$. Accordingly, all packets whose waiting time $D_{Q}$ has exceeded $D_{Q \max }$ are deleted to release space in the queue.

\section{PASS: Packet Aggregator/Scheduling System}

The PASS module (described in Algorithm 3) has been designed to optimize the data transmission in $\mathrm{EH}$ conditions. Its main objective is to determine the optimum number of data packets $\left(N_{t x}\right)$ in each transmission, which depends on the available energy $\left(E_{\text {stored }}\right)$ and the status of the data 


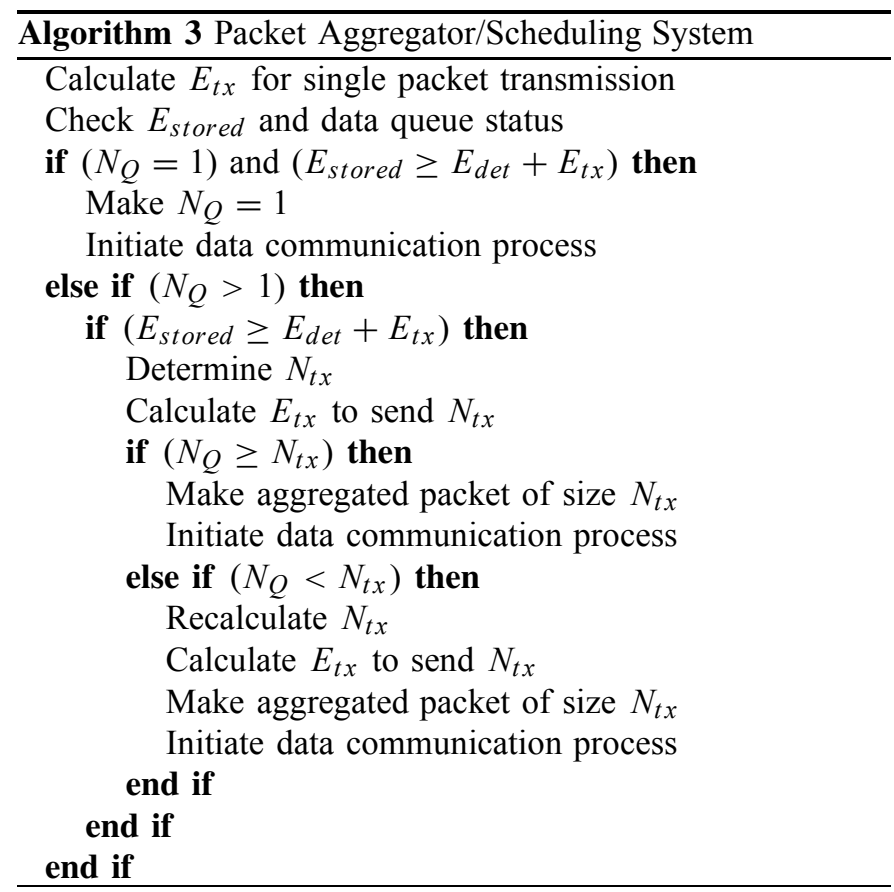

queue $\left(N_{Q}\right)$. Therefore, the value of $N_{t x}$ is indirectly adapted to the $\mathrm{EH}$ rate $K_{E H}$ and the packet arrival time at the BN. PASS also employs information of the IEEE 802.15.6 MAC protocol for the calculation of $E_{t x}$, which is also passed to the PHAM module. It should be emphasized that the selection of $N_{t x}$ is very critical, since a high $N_{t x}$ value would result to an increase of required transmission energy $E_{t x}$, whereas a low value would lead to inefficien performance.

\section{Performance Evaluation}

\section{A. Simulation Consideration and Setup}

We have developed an event-driven MATLAB simulator that implements our algorithm in a HEH-WBAN formed by a $\mathrm{BNC}$ and one $\mathrm{BN} .^{3}$ For our experiments, we have chosen the electrocardiograph (ECG) sensor node, which is a critical medical sensor that monitors the electrical activity of the heart. Events detected by the ECG are converted into packets and stored in a data buffer before their transmission. The QoS requirements [21] and the characteristics [18], [19] of the ECG are summarized in Table I, while the network parameters, which have been selected according to the IEEE 802.15.6 PHY-MAC specif cations [20], are summarized in Table II. ${ }^{4}$

We assume that the BNC has an external power supply (i.e., no energy shortage problems), while the $\mathrm{BN}$ is connected to a piezoelectric energy harvester that provides energy at a random rate $K_{E H}$, according to the body movements, and this energy is stored in a rechargeable supercapacitor. In addition, we consider four different human activities (i.e., relaxing, walking, running and cycling) with different $P_{E H_{\min }}$ and $P_{E H_{\max }}$ values, as well as different steady state probabilities (i.e., $\mu_{O N}$ and $\left.\mu_{O F F}\right)$ [13]. In active slots, the harvested power

\footnotetext{
${ }^{3}$ Please note that our scheme is designed to improve the individual performance of each $\mathrm{BN}$, regardless of the total number of BNs in the WBAN.

${ }^{4}$ In Table II, PLCP and FCS stand for Physical Layer Convergence Procedure and Frame Check Sequence, respectively.
}

TABLE I

ECG BN CHARACTERISTICS

\begin{tabular}{|c|c|c|c|}
\hline \multirow{3}{*}{$\begin{array}{c}\text { Data and } \\
\text { Traffic Features }\end{array}$} & \multicolumn{2}{|c|}{ Packet arrival time } & $2 \mathrm{~ms}$ \\
\hline & \multicolumn{2}{|c|}{ Data queue size } & 200 packets \\
\hline & \multicolumn{2}{|c|}{ Packet size } & 12 bits \\
\hline \multirow{6}{*}{$\begin{array}{c}\text { Power } \\
\text { Consumption } \\
\text { Distribution }\end{array}$} & \multicolumn{2}{|c|}{ Sensor READ-OUT and ADC } & $30 \mu W$ \\
\hline & \multicolumn{2}{|c|}{$\mathrm{MCU}$} & $19.25 \mu W$ \\
\hline & \multirow{4}{*}{ Transceiver } & Reception & $3.85 \mathrm{~mW}$ \\
\hline & & Transmission & $4.6 \mathrm{~mW}$ \\
\hline & & Idle & $0.712 \mathrm{~mW}$ \\
\hline & & Sleep & $4 \mu W$ \\
\hline \multirow{2}{*}{ QoS Requirements } & \multicolumn{2}{|c|}{ Delay Constraint } & $<250 \mathrm{~ms}$ \\
\hline & \multicolumn{2}{|c|}{ Packet Loss Constraint } & $<10 \%$ \\
\hline
\end{tabular}

TABLE II

Simulation PARAMETERS

\begin{tabular}{|c|c||c|c|}
\hline Parameter & Value & Parameter & Value \\
\hline Simulation Time & $60 \mathrm{~s}$ & MAC Header & 56 bits \\
\hline$p_{S I F S}$ & $0.05 \mathrm{~ms}$ & FCS & 16 bits \\
\hline$p_{C S M A}$ slot & $0.125 \mathrm{~ms}$ & PLCP Preamble & 90 bits \\
\hline PLCP Tx rate & $91.9 \mathrm{~kb} / \mathrm{s}$ & PLCP Header & 31 bits \\
\hline Data Tx rate & $485.7 \mathrm{~kb} / \mathrm{s}$ & ACK & 72 bits \\
\hline Control Tx rate & $121.4 \mathrm{~kb} / \mathrm{s}$ & $T_{P O L L}$ & 88 bits \\
\hline
\end{tabular}

TABLE III

EH SOURCE PARAMETERS

\begin{tabular}{|c|c|c|c|}
\hline Activity & $P_{E H \min }$ & $P_{E H \max }$ & $\mu_{O N}$ \\
\hline RELAXING & $1 \mu \mathrm{W}$ & $4.8 \mu \mathrm{W}$ & 0.9 \\
\hline WALKING & $128.6 \mu \mathrm{W}$ & $186 \mu \mathrm{W}$ & 0.1 \\
\hline RUNNING & $724.2 \mu \mathrm{W}$ & $910 \mu \mathrm{W}$ & $0.1-0.2$ \\
\hline CYCLING & $37.4 \mu \mathrm{W}$ & $72.3 \mu \mathrm{W}$ & 0.9 \\
\hline
\end{tabular}

is randomly selected in the range $\left[P_{E H_{\min }}, P_{E H_{\max }}\right]$. The parameters that characterize the energy harvesting source in each activity are summarized in Table III. As we can see, the probability of harvesting energy in relaxing and cycling modes is higher than the energy harvested during other activities, such as walking and running, as the specifi activities are continuous and not intermittent. However, the amount of energy that is harvested varies significantl, as the energy produced in relaxing mode due to the mild body movements is considerably low, unlike the energy produced during other activities (especially during running) which is relatively high due to their intensity. The aforementioned tradeoffs (i.e., between the probability of harvesting energy and the amount of harvested energy) make the study particularly interesting.

To evaluate our approach, we study the performance of an ECG with and without PEH-QoS, with respect to the following metrics: i) detection eff ciency, def ned as the number of detected events over the total number of occurred events; ii) data storage eff ciency, def ned as the number of stored events over the total number of detected events; iii) normalized throughput, defi ed as the number of successfully transmitted bits over the total number of generated bits, within the same period of time; iv) packet loss, def ned as the percentage of data packets that have not been received by the BNC (i.e., events that could not be detected due to the lack of energy, packets that could not be stored due to the saturation of the data queue, and stored packets that were not transmitted due to time restrictions); v) average packet end-to-end delay, def ned as the total time between the packet generation and the packet 


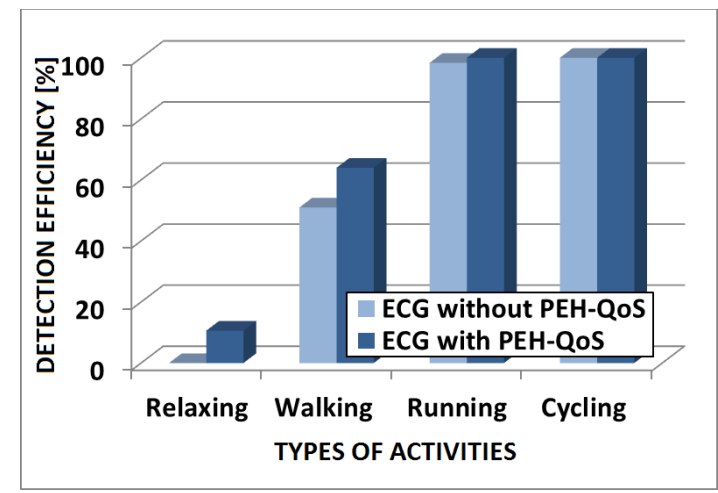

Fig. 4. Detection eff ciency for various types of human activities.

reception by the BNC; and vi) energy eff ciency, def ned as the total transmitted bits over the total energy consumption.

\section{B. Simulation Results}

Figure 4 presents the simulation results for the detection eff ciency of ECG with and without PEH-QoS. In the case of the relaxing activity, we can see that the PEH-QoS improves the detection eff ciency of the ECG by $10.6 \%$. More specifcally, although movements occur during $90 \%$ of the total time (i.e., $\mu_{O N}=0.9$ ), the ECG without the PEH-QoS can hardly detect any events, mainly due to the very small amount of energy that is collected (i.e., $1 \mu W \leq K_{E H} \leq 4.8 \mu \mathrm{W}$ ). As the ECG without PEH-QoS is not aware of the available energy, it persistently tries to detect events when there is not suff cient energy to complete this process. As a result, events are not detected successfully, whereas the collected energy is wasted. On the contrary, our system makes better use of the available energy, since the BN waits until there is sufficien accumulated energy to guarantee a successful detection. In the same f gure, we can also observe another interesting behavior related to the walking activity. Let us recall that, in this activity, the power values captured by the node are much higher compared to the relaxing mode (i.e., $128.6 \mu \mathrm{W} \leq K_{E H} \leq 186 \mu \mathrm{W}$ ), but the availability of the source is only $10 \%$. However, under these conditions, the node can obtain suff cient amount of energy to detect events, as the baseline approach reaches $51 \%$, while our system achieves $64 \%$ of detection eff ciency, by enhancing the use of the harvested energy. With regard to running and cycling activities, the generated energy is high enough to permit almost $100 \%$ detection eff ciency either with or without PEH-QoS. In the case of cycling, even though the captured power range $\left(37.4 \mu \mathrm{W} \leq K_{E H} \leq 72.3 \mu \mathrm{W}\right)$ is much lower with respect to running $\left(724.2 \mu \mathrm{W} \leq K_{E H} \leq 910 \mu \mathrm{W}\right)$, the availability of the source is significa tly high $(90 \%)$, allowing the node to gain suff cient energy levels to perform proper detection. In the case of running, the harvested power level is so high that $100 \%$ detection is achieved even though the source is available only $10 \%$ of the time.

Figure 5 shows the behavior of the data queue during the execution of the running activity. At the beginning of the experiment (i.e., $t=0 \mathrm{~ms}$ ), the battery of the node is empty and, therefore, packets are accumulated at the node's buffer until the harvested energy reaches the required level to

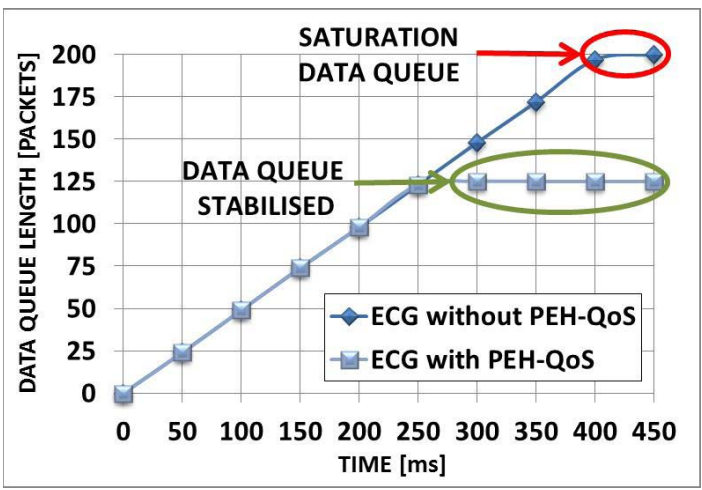

Fig. 5. Data queue length versus time for the running activity.

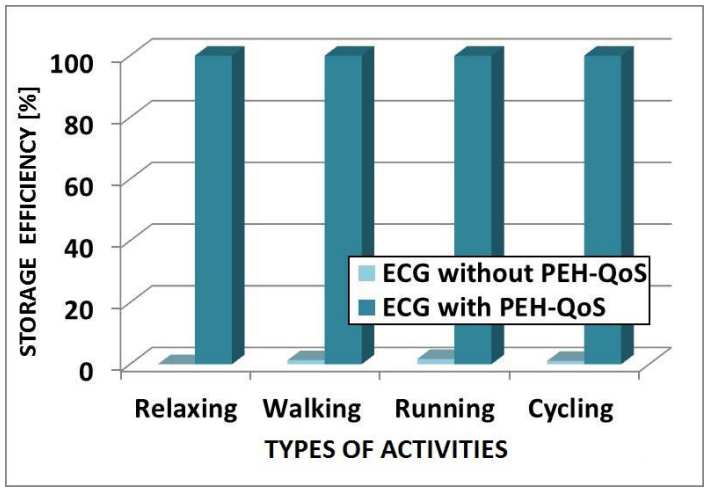

Fig. 6. Storage eff ciency for various types of human activities.

perform data transmissions. Under these conditions, the queue of the baseline scheme saturates and any new packets are dropped due to lack of storage space. However, by applying the PEH-QoS algorithm, the queue is stabilized thanks to the eff cient management of the available energy and the effective elimination of the useless data packets. More specificall , the queue is stabilized at 124 data packets, where $100 \%$ of the stored information is valid. Accordingly, it can be concluded that the optimal aggregation factor in this case is $N_{t x}=124$.

Figure 6 demonstrates the storage eff ciency of the two schemes (PEH-QoS and baseline) for the four different activities. As we can see, in case of the baseline scenario, most of the detected events cannot be stored, as the queue saturates since there is not enough energy for the data packet transmission. For better performance, a higher EH rate $K_{E H}$ would be required, which is not feasible with current technologies. On the other hand, PEH-QoS maintains the storage eff ciency at $100 \%$ thanks to the eff cient data queue management.

Figure 7 shows the normalized throughput in our scheme for different aggregation size $\left(N_{t x}\right)$ values during the execution of the running activity for two different availability cases (i.e., $\mu_{O N}=0.1$ and $\mu_{O N}=0.2$ ). In the frst case (Figure 7), it can be seen that $N_{t x}=124$ achieves the highest normalized throughput (59\%), thus conf rming that this is the optimum value for data packets transmission in these conditions. In the second case (Figure 7), as expected, the increase of the availability of the source increases the normalized throughput, reaching $100 \%$ for $N_{t x}=75,100$ and 124 . Consequently, these values can be considered as optimal in the aggregation system. 


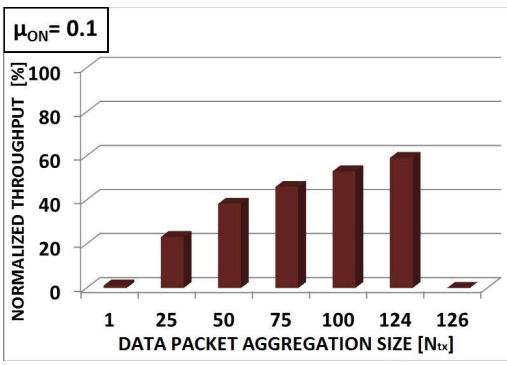

(a)

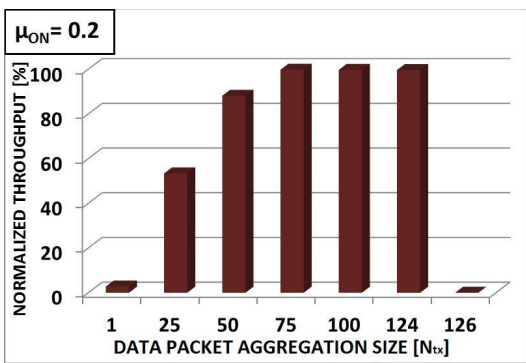

(b)

Fig. 7. Normalized throughput versus packet aggregation size $\left(N_{t x}\right)$ for the running activity. (a) Case I $\left(\mu_{O N}=0.1\right)$. (b) Case II $\left(\mu_{O N}=0.2\right)$.

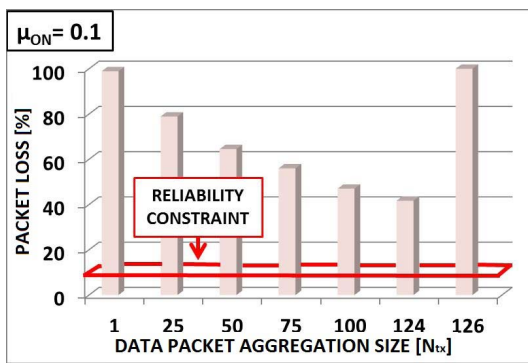

(a)

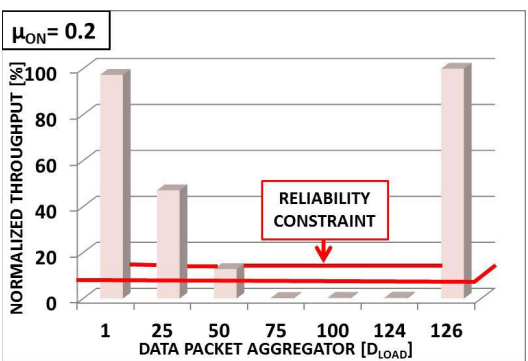

(b)

Fig. 8. Packet loss versus packet aggregation size $\left(N_{t x}\right)$ for the running activity. (a) Case I $\left(\mu_{O N}=0.1\right)$. (b) Case II $\left(\mu_{O N}=0.2\right)$.

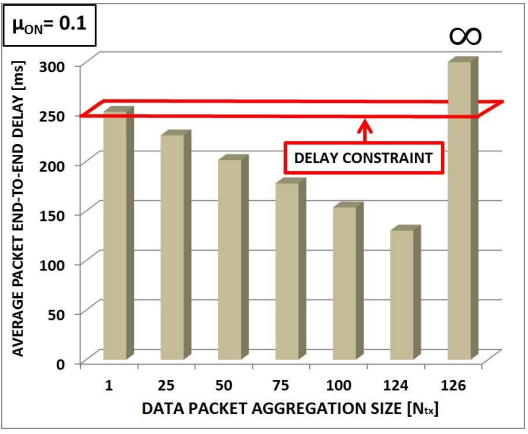

(a)

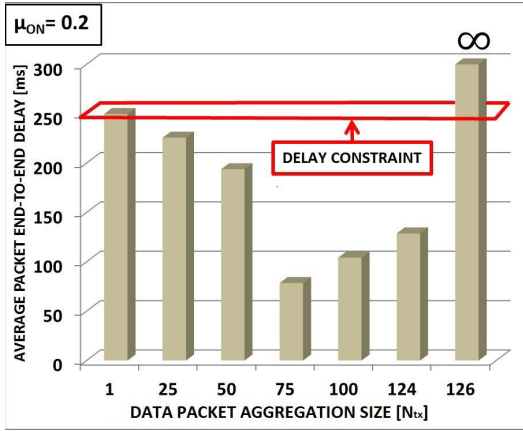

(b)

Fig. 9. Packet delay versus packet aggregation size $\left(N_{t x}\right)$ for the running activity. (a) Case I $\left(\mu_{O N}=0.1\right)$. (b) Case II $\left(\mu_{O N}=0.2\right)$.

For the same conditions, Figures 8 and 9 show the relationship between $N_{t x}$ and the restrictions of reliability and delay, respectively. As it can be seen in Figure 8, none of the $N_{t x}$ values meets the reliability constraint, as the harvested energy level is too low, causing a percentage of lost packets that exceeds the permitted levels. On the other hand, when $\mu_{O N}=0.2$ (Figure 8), the optimal $N_{t x}$ values (i.e., 75,100 , and 124) fulfil the reliability constraint, keeping the packet loss in acceptable levels. Regarding the packet delay, as it can be observed in Figure 9, the delay constraint is met in both cases (i.e., $\mu_{O N}=0.1$ and $\mu_{O N}=0.2$ ).

In Figure 10, we study the energy eff ciency of the node in the running mode, assuming two different availability probabilities and two different values for the packet aggregation. In this figure we can observe that the energy eff ciency increases, as the number of aggregated packet grows. In particular, PEH-QoS achieves up to 56 and 51 times higher energy

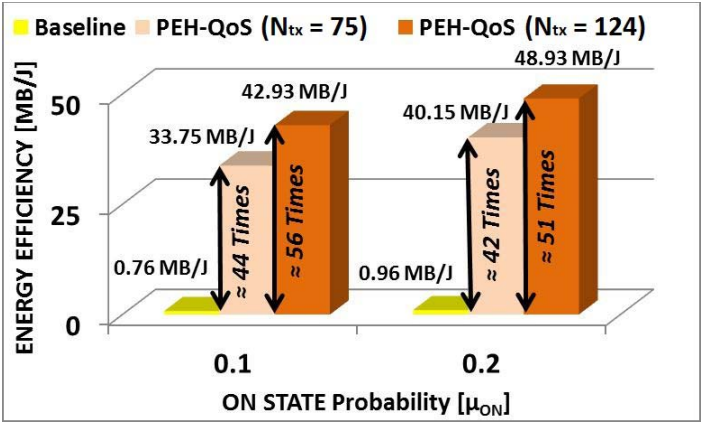

Fig. 10. Energy efficien y for the running activity.

efficien y from the baseline scenario in case of $\mu_{O N}=0.1$ and $\mu_{O N}=0.2$, respectively. This fact can be rationally explained by considering that, in case of the ECG with PEH-QoS $\left(N_{t x}=124\right)$, the node spends only $E_{t x}=24.3 \mu \mathrm{J}$ 


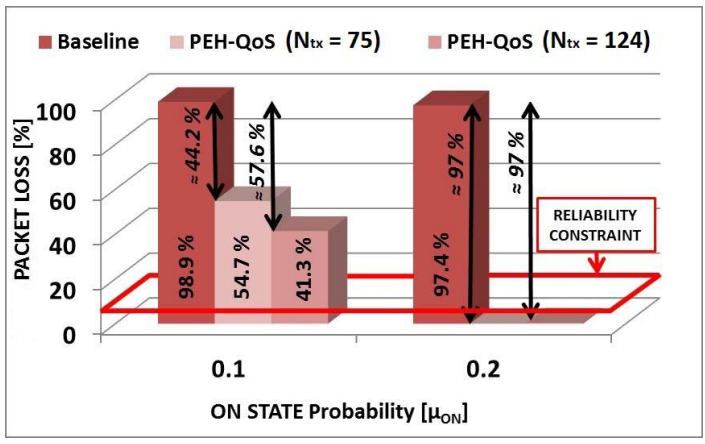

Fig. 11. Packet loss for the running activity.

for the transmission of 124 data packets of 12 bits. On the other hand, in the case of simple nodes, where no aggregation is applied (i.e., $N_{t x}=1$ ), the amount of $E_{t x}=10.3 \mu \mathrm{J}$ is required for the transmission of a single data packet of 12 bits.

Finally, in Figure 11, we study the packet loss in the same scenario, where our scheme fulfill the reliability required by the application (i.e., maximum packet loss $10 \%$ ) only in case of $\mu_{O N}=0.2$, while the baseline system exceeds this threshold in both energy harvesting conditions $\left(\mu_{O N}=0.1\right.$ and $\mu_{O N}=0.2$ ). In particular, for $\mu_{O N}=0.2$, our system achieves $0.38 \%$ packet loss, while the baseline reaches $97.4 \%$ packet loss.

\section{CONCLUding REMARKS}

In this paper, we introduced PEH-QoS, a novel and highly eff cient control scheme for EH-powered BNs. The proposed QoS-aware control scheme has a modular architecture that enables the optimal use of the energy collected in the human environment. Extensive simulations have shown that the application of PEH-QoS signif cantly improves both the transmission and the detection of the medical events, increasing the normalized throughput and the energy eff ciency, while reducing the packet loss and the average packet end-to-end delay. In our future work, we plan to analytically evaluate the performance of the proposed framework.

\section{REFERENCES}

[1] P. A. Gould and A. D. Krahn, "Complications associated with implantable cardioverter-def brillator replacement in response to device advisories," J. Amer. Med. Assoc., vol. 295, no. 16, pp. 1907-1911, Apr. 2006.

[2] Y.-T. Lin, Y.-S. Lin, C.-H. Chen, H.-C. Chen, Y.-C. Yang, and S.-S. Lu, "A 0.5-V biomedical system-on-a-chip for intrabody communication system," IEEE Trans. Ind. Electron., vol. 58, no. 2, pp. 690-699, Feb. 2011.

[3] J. A. Paradiso and T. Starner, "Energy scavenging for mobile and wireless electronics," IEEE Pervasive Comput., vol. 4, no. 1, pp. 18-27, Jan./Mar. 2005.

[4] Y. K. Tan and S. K. Panda, "Energy harvesting from hybrid indoor ambient light and thermal energy sources for enhanced performance of wireless sensor nodes," IEEE Trans. Ind. Electron., vol. 58, no. 9, pp. 4424-4435, Sep. 2011

[5] A. Kansal, J. Hsu, S. Zahedi, and M. B. Srivastava, "Power management in energy harvesting sensor networks," ACM/IEEE Trans. Embedded Comput. Syst., vol. 6, no. 4, pp. 651-656, Sep. 2007.

[6] A. Khaligh, P. Zeng, and C. Zheng, "Kinetic energy harvesting using piezoelectric and electromagnetic technologies - State of the art," IEEE Trans. Ind. Electron., vol. 57, no. 3, pp. 850-860, Mar. 2010.
[7] V. Goudar, Z. Ren, P. Brochu, M. Potkonjak, and Q. Pei, "Optimizing the output of a human-powered energy harvesting system with miniaturization and integrated control," IEEE Sensors J., vol. 14, no. 7, pp. 2084-2091, Jul. 2014.

[8] P. Li, Y. Wen, C. Jia, and X. Li, "A magnetoelectric composite energy harvester and power management circuit," IEEE Trans. Ind. Electron., vol. 58, no. 7, pp. 2944-2951, Jul. 2011.

[9] P. Li, Y. Wen, W. Yin, and H. Wu, "An upconversion management circuit for low-frequency vibrating energy harvesting," IEEE Trans. Ind. Electron., vol. 61, no. 7, pp. 3349-3358, Jul. 2014.

[10] J. Colomer-Farrarons, P. Miribel-Catala, A. Saiz-Vela, and J. Samitier, "A multiharvested self-powered system in a low-voltage low-power technology," IEEE Trans. Ind. Electron., vol. 58, no. 9, pp. 4250-4263, Sep. 2011.

[11] P. Spachos and D. Hantzinakos, "Scalable dynamic routing protocol for cognitive radio sensor networks," IEEE Sensors J., vol. 14, no. 7, pp. 2257-2266, Jul. 2014.

[12] P. Spachos, D. Toumpakaris, and D. Hatzinakos, "QoS and energy-aware dynamic routing in wireless multimedia sensor networks," in Proc. IEEE ICC, London, U.K., Jun. 2015, pp. 6935-6940.

[13] A. Seyedi and B. Sikdar, "Modeling and analysis of energy harvesting nodes in body sensor networks," in Proc. 5th Int. Summer School Symp. Med. Devices Biosensors (ISSS-MDBS), Jun. 2008, pp. 175-178.

[14] A. Seyedi and B. Sikdar, "Energy eff cient transmission strategies for body sensor networks with energy harvesting," IEEE Trans. Commun., vol. 58, no. 7, pp. 2116-2126, Jul. 2010.

[15] J. Ventura and K. Chowdhury, "Markov modeling of energy harvesting body sensor networks," in Proc. IEEE 22nd Int. Symp. Pers. Indoor Mobile Radio Commun. (PIMRC), Sep. 2011, pp. 2168-2172.

[16] Y. He, W. Zhu, and L. Guan, "Optimal resource allocation for pervasive health monitoring systems with body sensor networks," IEEE Trans. Mobile Comput., vol. 10, no. 11, pp. 1558-1575, Nov. 2011.

[17] S. Sudevalayam and P. Kulkarni, "Energy harvesting sensor nodes: Survey and implications," IEEE Commun. Surveys Tuts., vol. 13, no. 3, pp. 443-461, Jan. 2011

[18] Y.-H. Liu et al., "A 1.9 nJ/b $2.4 \quad \mathrm{GHz}$ multistandard (Bluetooth low energy/Zigbee/IEEE802.15.6) transceiver for personal/body-area networks," in IEEE Int. Solid-State Circuits Conf. Dig. Tech. Papers (ISSCC), Feb. 2013, pp. 446-447.

[19] R. F. Yazicioglu, S. Kim, T. Torfs, P. Merken, and C. Van Hoof, "A $30 \mu \mathrm{W}$ analog signal processor ASIC for biomedical signal monitoring," in IEEE Int. Solid-State Circuits Conf. Dig. Tech. Papers (ISSCC), Feb. 2010, pp. 124-125.

[20] Standard for Local and Metropolitan Area Networks-Part 15.6: Wireless Body Area Networks, IEEE Standard 802.15.6-2012, Feb. 2012.

[21] M. A. Razzaque, C. S. Hong, and S. Lee, "Data-centric multiobjective QoS-aware routing protocol for body sensor networks," Sensors, vol. 11, no. 1, pp. 917-937, Jan. 2011.

Ernesto Ibarra received the Ph.D. degree from the University of Barcelona in 2014. His main research interests include energy harvesting, body sensor networks, and human health.

Angelos Antonopoulos received the Ph.D. degree from the Technical University of Catalonia in 2012. He is currently a Post-Doctoral Researcher. His main research interests include radio resource management and energy efficien network planning.

Elli Kartsakli received the Ph.D. degree in wireless communications from the Technical University of Catalonia (UPC) in 2012. She is currently a Post-Doctoral Researcher with UPC. She has participated in several national and European projects.

Joel J. P. C. Rodrigues is currently a Professor with the Department of Informatics, University of Beira Interior, Covilhã, Portugal, and a Senior Researcher with the Instituto de Telecomunicações, Portugal. He is the Leader of the NetGNA Research Group and the Chair of the IEEE ComSoc Technical Committee on eHealth.

Christos Verikoukis received the Ph.D. degree from the Technical University of Catalonia in 2000. He is currently a Senior Researcher and the Head of the SMARTECH Department with CTTC and an Adjunct Associate Professor with the University of Barcelona. 